Revista Brasileira de Agricultura Irrigada v.12, nº.5, p. 2976 - 2983, 2018

ISSN 1982-7679 (On-line)

Fortaleza, CE, INOVAGRI - http://www.inovagri.org.br

DOI: $10.7127 /$ rbai.v12n500946

Protocolo 946.18 - 21/05/2018 Aprovado em 04/09/2018

\title{
DESEMPENHO AGRONÔMICO DA CULTURA DO MILHO SOB DIFERENTES ARRANJOS ESPACIAIS NO NORDESTE BRASILEIRO
}

\author{
Valberto Rômulo Feitosa Pereira ${ }^{1}$, Carlos Alessandro Chioderoli ${ }^{2}$, Daniel Albiero ${ }^{3}$, \\ Alexsandro Oliveira da Silva ${ }^{4}$, Elivania Maria Sousa Nascimento ${ }^{5}$, Paulo Ricardo Alves dos \\ Santos ${ }^{6}$
}

\section{RESUMO}

O objetivo com este trabalho foi avaliar o desempenho agronômico do milho em função da população de plantas e espaçamentos entre linhas em um Argissolo Vermelho Amarelo no nordeste brasileiro. $\mathrm{O}$ experimento foi realizado em área pertencente à Universidade Federal do Ceará, Fortaleza - CE, com delineamento em blocos casualizados no esquema fatorial $2 \times 2$, com 20 repetições, sendo os tratamentos constituídos por duas populações (45.000 e 65.000 plantas ha-1) e dois espaçamentos $(0,45$ e $0,90 \mathrm{~m})$. Avaliou-se a produção por hectare, número de espigas por hectare, plantas por hectare, altura da planta, altura de inserção da $1^{\mathrm{a}}$ espiga, massa seca total, número de grãos por fileiras, número de fileiras, emergência e diâmetro do colmo. Observou-se diferenças significativas entre a população e o espaçamento para a produção por hectare, espigas por hectare, plantas por hectare e emergência. A população de 65.000 plantas ha ${ }^{-1}$ no espaçamento de $0,90 \mathrm{~m}$ proporcionou incremento na produção por hectare, espiga por hectare e emergência, quando comparadas à população de 45.000 plantas $\mathrm{ha}^{-1}$ e espaçamentos entre linhas de $0,45 \mathrm{~m}$.

Palavras-chave: Zea Mays, produtividade, manejo.

\section{AGRONOMIC PERFORMANCE OF CORN CULTURE UNDER DIFFERENT SPACE ARRANGEMENTS IN NORTHEAST BRAZIL}

\footnotetext{
${ }^{1}$ Instituto Federal de Educação Básica e Tecnológica, IFCE, Departamento de Física e Matemática, Fortaleza, CE, Brasil, email: valbertofeitosa@uol.com.br.

${ }^{2}$ Universidade Federal do Triângulo Mineiro, UFTM, Departamento de Agronomia, Iturama, MG, Brasil, email: ca.chioderoli@gmail.com.

${ }^{3}$ Universidade Estadual de Campinas, UNICAMP, Campinas, SP, Brasil, dalbiero@ufc.br.

${ }^{4}$ Universidade Federal do Ceará, UFC, Departamento de Engenharia Agrícola, Fortaleza, CE, Brasil, email: alexsandro@ufc.br.

${ }^{5}$ Universidade Federal do Ceará, UFC, Departamento de Engenharia Agrícola, Fortaleza, CE, Brasil, email: elivania_sousa@yahoo.com.br.

${ }^{6}$ Universidade Federal do Ceará, UFC, Departamento de Engenharia Agrícola, Fortaleza, CE, Brasil, email: paulo_ptg@hotmail.com.
} 


\begin{abstract}
The objective of this work was to evaluate the agronomic performance of maize as a function of the plant population and line spacings, in a Red Yellow Argisol in northeast Brazil. The experiment was carried out in an area belonging to the Federal University of Ceará, Fortaleza, Brazil, with a randomized complete block design in the $2 \times 2$ factorial scheme, with 20 replications, with two populations (45,000 and 65,000 plants ha $\left.{ }^{-1}\right)$ and two spacings $(0.45$ and $0.90 \mathrm{~m}$ ). It was evaluated the yield per hectare, number of spikes per hectare, plants per hectare, height of the plant, height of insertion of the first spike, total dry mass, number of grains per row, number of rows, emergence and stem diameter. Significant differences were observed between population and spacing for yield per hectare, spikes per hectare, plants per hectare, and emergence. The population of 65,000 plants $^{-1} \mathrm{~h}^{-1}$ in the $0.90 \mathrm{~m}$ spacing provided an increase in yield per hectare, spike per hectare and emergence, when compared to the population of 45,000 $\mathrm{ha}^{-1}$ plants and row spacings of $0.45 \mathrm{~m}$.
\end{abstract}

Keywords: Zea Mays, productive, management.

\section{INTRODUÇÃO}

O milho (Zea mays L.) é um dos cereais mais cultivados no mundo. No Brasil, a área cultivada com essa cultura foi de 10,5 milhões de hectares, com uma produção de 40,7 milhões de toneladas com produtividade média de 4.818 $\mathrm{kg} \mathrm{ha}^{-1}$ (CONAB 2016).

A cultura tem papel socioeconômico importante no agronegócio brasileiro devido sua produção estar inserida em todas as regiões no país. No entanto, a produtividade do milho no Brasil é considerada baixo quando comparada ao Estado Unidos que apresenta média produtividade acima de $10.000 \mathrm{~kg} \mathrm{ha}^{-1}$. Tal fato pode estar relacionado às condições climáticas, fertilidade do solo, uso eficiente de corretivos e fertilizantes, e na escolha de cultivares mais adaptadas para a região (Sangoi et al., 2010; Chioderoli et al. 2012).

Entre as tecnologias utilizadas na cultura do milho, ressalta-se o sistema plantio direto com o objetivo de reduzir custos de produção, atrelado à necessidade de melhoria na qualidade do solo visando uma produção sustentável (Albuquerque et al. 2013). Alterações na densidade de plantas, por meio do espaçamento entre linhas e da distribuição de plantas na linha de cultivo, proporcionam diferentes arranjos, e vem sendo foco de estudo de vários pesquisadores nacionalmente.

A população de plantas é um dos principais fatores que influem na capacidade da lavoura em captar recursos do ambiente (água, luz e nutrientes). Silva et al. (2014) objetivando avaliar o efeito da população de plantas sobre o crescimento e a produtividade de dois híbridos de milho, concluíram que as densidades de 60.000 e 80.000 plantas ha ${ }^{-1}$ proporcionam incrementos na produtividade de grãos de 12,5 e $13,6 \%$, respectivamente, quando comparadas à população de 40.000 plantas ha ${ }^{-1}$.

O espaçamento entre fileiras da lavoura, por sua vez, guarda forte relação com o arranjo espacial entre plantas, sendo passível de ajuste com o intuito principal de reduzir a competição intraespecífica Foloni et al. (2014). A cultura do milho é tradicionalmente implantada no Brasil com espaçamentos entre linhas de 0,80 e 0,90 m, em sistema convencional. Hoffmann et al. (2015) avaliando a produção de milho em diferentes espaçamentos, concluíram que o melhor espaçamento a ser utilizado é o de 0,90 $\mathrm{m}$, pois oferece menor competição entre as plantas.

Portanto, se faz justificável a condução de pesquisas que busquem a melhor população de plantas e espaçamentos que tragam maiores produtividades para a cultura do milho, bem como, conhecer o comportamento da cultura frente a esses fatores (população $\mathrm{x}$ espaçamentos) para cada região. Assim, o objetivo com este trabalho foi avaliar o desempenho agronômico da cultura do milho em função da população de plantas e espaçamentos 
entre linhas, em um Argissolo Vermelho Amarelo no nordeste brasileiro.

\section{MATERIAL E MÉTODOS}

O trabalho foi conduzido em área experimental pertencente ao Departamento de Engenharia Agrícola, da Universidade Federal do Ceará, em Argissolo Vermelho Amarelo (EMBRAPA, 2013). A área experimental está situada nas coordenadas geográficas de

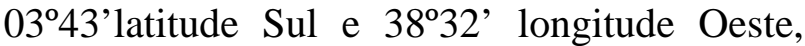
situada a $19 \mathrm{~m}$ de altitude. O clima da região apresenta-se como tropical chuvoso do tipo Aw' conforme classificação de Koppen. Os dados metereológicos do período de condução do experimento encontra-se na tabela 1 .

Tabela 1. Dados meteorológicos relativos ao período de condução do experimento (Maio a Setembro de 2016)

\begin{tabular}{ccccc}
\hline Meses & $\begin{array}{c}\text { Precipitação } \\
(\mathrm{mm})\end{array}$ & $\begin{array}{c}\text { Temperatura } \\
\text { mínima } \\
\left({ }^{0} \mathrm{C}\right)\end{array}$ & $\begin{array}{c}\text { Temperatura } \\
\text { média } \\
\left({ }^{0} \mathrm{C}\right)\end{array}$ & $\begin{array}{c}\text { Temperatura } \\
\text { máxima } \\
\left({ }^{0} \mathrm{C}\right)\end{array}$ \\
\hline Maio & 160,1 & 23,7 & 28,7 & 30,2 \\
Junho & 53,6 & 22,6 & 28,9 & 31,6 \\
Julho & 17 & 21,9 & 28,7 & 31,2 \\
Agosto & 4,7 & 21,9 & 28,5 & 31,1 \\
Setembro & 5,8 & 22,5 & 28,1 & 31,2 \\
\hline
\end{tabular}

milímetros; ${ }^{0} \mathrm{C}$ - grau celsius

Antes da instalação do experimento foram coletadas amostras de solo para caracterização química nas camadas de 0-0,15 e 0,15-0,30 m
(Tabela 2), seguindo a metodologia da (EMBRAPA, 2011).

Tabela 2. Características químicas iniciais do solo da área experimental

\begin{tabular}{|c|c|c|c|c|c|c|c|c|c|c|c|}
\hline \multirow{2}{*}{$\begin{array}{l}\text { Prof. } \\
\text { (cm) }\end{array}$} & \multirow{2}{*}{$\begin{array}{c}\mathrm{pH} \\
\left(\mathrm{H}_{2} \mathrm{O}\right)\end{array}$} & $\mathrm{Ca}^{2+}$ & $\mathrm{Mg}^{2+}$ & $\mathrm{Na}^{+}$ & $\mathrm{K}^{+}$ & $\mathrm{H}^{+}+\mathrm{Al}^{3+}$ & $\mathrm{Al}^{3+}$ & $\mathrm{S}$ & $\mathrm{T}$ & \multirow{2}{*}{$\begin{array}{c}\mathrm{V} \\
(\%)\end{array}$} & \multirow{2}{*}{$\begin{array}{c}\mathrm{m} \\
(\%)\end{array}$} \\
\hline & & \multicolumn{8}{|c|}{$\mathrm{mmol}_{\mathrm{c}} \mathrm{dm}^{-3}$} & & \\
\hline $0-15$ & 5,5 & 14 & 10 & 15 & 14 & 14,9 & 0,10 & 28 & 30 & 65 & 30 \\
\hline $15-30$ & 5,2 & 13 & 0,7 & 12 & 10 & 16,5 & 0,10 & 22 & 29 & 57 & 40 \\
\hline
\end{tabular}

S: soma de bases; T: capacidade de troca de cátions potencial; V: saturação por bases; m saturação por alumínio

O delineamento usado no experimento foi em blocos casualizado, em esquema fatorial $(2 \mathrm{x}$ 2), constituído de duas populações (P1 - 45.000 plantas ha-1 ${ }^{-1}$ P2 - 65.000 plantas $\left.h^{-1}\right)$ e dois espaçamentos (E1 - 0,45 m; E2 - 0,90 m), com vinte repetições.

A semeadura do milho foi realizada manualmente, para isso utilizou-se a cultivar $\mathrm{Al}$ Avaré, considerado de baixa a alta tecnologia, com pureza de $98 \%$ e sobrevivência de $85 \%$. A irrigação foi realizada por aspersão convencional, sendo calculado o tempo de irrigação de acordo com os estádios fenológico da cultura, os quais foram: inicial $(0,30$ - 0,50); vegetativo $(0,80$ - 0,85$)$; produção $(1,05-1,20)$; maturação $(0,95-1,05)$ e colheita $(0,55-0,60)$ (DOORENBOS \& PRUITT, 1977).
Foram realizadas adubações de base e de cobertura baseadas na análise química do solo; na adubação de base utilizou-se $250 \mathrm{~kg} \mathrm{ha}^{-1}$ de fertilizante na formulação comercial de NPK 1028-20. A adubação de cobertura foi realizada nos estádios $\mathrm{V}_{2}, \mathrm{~V}_{4}$ e $\mathrm{V}_{8}$ do milho, utilizando $300 \mathrm{~kg} \mathrm{ha}^{-1}$ de ureia e $120 \mathrm{~kg} \mathrm{ha}^{-1}$ de cloreto de potássio. Para controlar a presença da lagarta do cartucho (Spodoptera frugiperda) foram efetuadas quatro aplicações do produto Lufenuron (i.a) na dose de $18 \mathrm{~g} \mathrm{ha}^{-1}$ do ingrediente ativo e Lannat $\mathrm{BR}$ inseticida fosforado (princípio ativo: metomil), nos estádios $\mathrm{V}_{4}, \mathrm{~V}_{8}, \mathrm{~V}_{12}$ e $\mathrm{R}_{1}$.

Foram avaliadas características ligadas ao crescimento da planta e componentes de produção: (a) altura da planta - AP; (b) altura de 
inserção da $1^{\text {a }}$ espiga - AIE; (c) diâmetro do colmo - DC; (d) número de fileiras por espiga NFE; (e) número de grãos por fileiras - NGF; (f) índice de velocidade de emergência - IVE; (g) número de espiga por hectares - NEH; (h) matéria seca total - MST; (i) produção por hectares $-\mathrm{PH}$.

A altura de planta - (AP), consistiu na medição da distância do nível do solo a inserção da folha bandeira; a altura de inserção da $1^{\text {a }}$ espiga (AIE), foi obtida na medição da superfície do solo até a inserção da primeira espiga;

O diâmetro do colmo - (DC), foi obtida com medições no internódio situado acima do primeiro nó das raízes adventícias;

Número de fileiras por espiga - (NFE), foi calculado contando o número de fileiras por espigas;

Número de grãos por fileiras - (NGF), foi obtido contando-se o número de grãos por fileira por espigas;

Índice de velocidade de emergência (IVE), calculado a partir de contagens diárias conforme metodologia proposta por Maguire (1962);

Número de espiga por hectares - (NEH), calculados a partir da contagem das espigas presentes nas linhas centrais de cada parcela, sendo extrapolados os valores encontrados para número de espigas $\mathrm{ha}^{-1}$;

Matéria seca total - (MST), inicialmente foi realizado o corte das plantas de milho a $2 \mathrm{~cm}$ acima da superfície do solo dentro da área útil, posteriormente pesadas e descontado o peso dos grãos após a trilha, obtendo-se, assim, o peso da massa verde da palha. Em sequência, as amostras foram levadas a estufa com circulação forçada de ar, por $72 \mathrm{~h}$ a $65^{\circ} \mathrm{C}$, determinando-se a porcentagem de água e posterior cálculo da produção de massa seca de palha, em $\mathrm{kg} \mathrm{ha}^{-1}$;

Produção por hectares - (PH), calculada, após as espigas terem passada na trilha, sendo separados os grãos, pesados e determinada a umidade com valores corrigidos a 13\% de umidade, com base nas Regras de Análise de Sementes Brasil (BRASIL, 2009), após transformar a massa dos grãos à base de $13 \%$ os resultados foram extrapolados para $\mathrm{kg} \mathrm{ha}^{-1}$. Todas as avaliações foram realizadas em 10 plantas presente na área útil por parcela.

Os dados foram submetidos à análise de variância, após verificação da normalidade, ao nível de 5\% de significância e quando significativos, foi aplicado o teste de Tukey a $5 \%$ de probabilidade para comparação das médias. Para realização das análises, foi utilizado o programa estatístico Assistat 7.7 (Silva \& Azevedo, 2016).

\section{RESULTADOS E DISCUSSÃO}

De acordo com a tabela 2, não foi observada diferença significativa para as variáveis altura de inserção da $1^{\mathrm{a}}$ espiga, altura da planta e matéria seca total, porém para a variável diâmetro de colmo, houve interação entre os fatores (população de plantas $\mathrm{x}$ espaçamentos) (Tabela 3).

Tabela 3. Altura de planta (AP), altura de inserção de espiga (AIE), diâmetro de colmo (DC) e matéria seca total (MST) em função da população de plantas e espaçamento entrelinhas na cultura do milho

\begin{tabular}{ccccc}
\hline & $\begin{array}{c}\text { AP } \\
(\mathrm{cm})\end{array}$ & $\begin{array}{c}\text { AIE } \\
(\mathrm{cm})\end{array}$ & $\begin{array}{c}\text { DC } \\
(\mathrm{cm})\end{array}$ & $\begin{array}{c}\text { MST } \\
(\mathrm{kg})\end{array}$ \\
\hline População (P) & & & & \\
45.000 & 129,32 & 64,03 & 20,02 & 2,93 \\
65.000 & 124,87 & 61,58 & 19,24 & 2,80 \\
$\begin{array}{c}\text { Espaçamentos (E) } \\
0,45\end{array}$ & 126,51 & 63,65 & 19,86 & 3,05 \\
0,90 & 127,68 & 61,98 & 19,41 & 2,69 \\
\hline Teste F & & & & \\
\hline P & $1,74^{\mathrm{ns}}$ & $0,95^{\mathrm{ns}}$ & $1,64^{\mathrm{ns}}$ & $0,21^{\mathrm{ns}}$ \\
E & $0,12^{\mathrm{ns}}$ & $0,44^{\mathrm{ns}}$ & $0,53^{\mathrm{ns}}$ & $1,68^{\mathrm{ns}}$ \\
$\mathrm{P} \times \mathrm{E}$ & $0,35^{\mathrm{ns}}$ & $0,31^{\mathrm{ns}}$ & $8,91^{* *}$ & $0,66^{\mathrm{ns}}$ \\
\hline
\end{tabular}




\begin{tabular}{ccccc}
\hline DMS & & & & \\
\hline $\mathrm{P}$ & 6,70 & 5,00 & 1,22 & 0,55 \\
$\mathrm{E}$ & 6,70 & 5,00 & 1,22 & 0,55 \\
$\mathrm{CV}$ & 11,84 & 17,89 & 13,97 & 43,61 \\
\hline
\end{tabular}

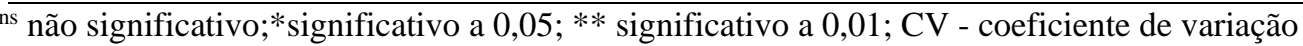

Trabalhando com população de 60, 70 e 80 mil plantas ha ${ }^{-1}$ Neumann et al. (2018) também observaram que a densidade populacional não alterou as variáveis altura de planta e inserção da primeira espiga. Já Fantin et al. (2016) verificaram que menores densidades de plantas reduzem a altura da planta e favorecem o engalhamento, devido à maior entrada de luz no dossel, o que não foi observado neste trabalho. Em espaçamentos mais aberto, o milho tem como característica apresentar maior altura de planta, por conseguinte, maior altura de inserção de espigas (Porto et al. 2011).

Em sua pesquisa com a cultura do milho no mesmo solo do presente trabalho, Santos et al. (2017) também não encontraram modificações nas características morfológicas do milho. Tais autores salientam que o estudo se

A.

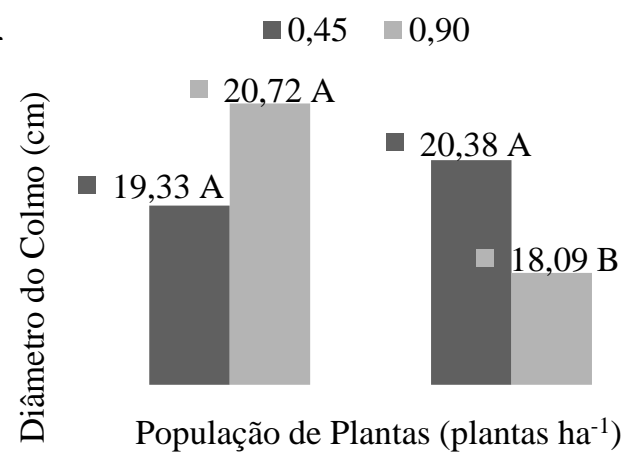

deu apenas em um ciclo, o que se faz necessárias avaliações com mais de um ciclo. Para a variável matéria seca total (MST), também não foi observado efeito significativo para os fatores (população e espaçamentos), nem tampouco, interação entre eles, concordando Klein et al. (2018).

Os valores do desdobramento para a variável diâmetro de colmo entre os tratamentos espaçamento e população de plantas constam na Figura 2A e 2B. Verifica-se que na população de 45.000 plantas por hectare, os espaçamentos não interferiram na alteração do diâmetro do colmo (DC), no entanto, para a população de 65.000 plantas por hectare, o diâmetro do colmo foi maior. Provavelmente esses resultados podem estar associados com o maior aproveitamento dos recursos do meio.

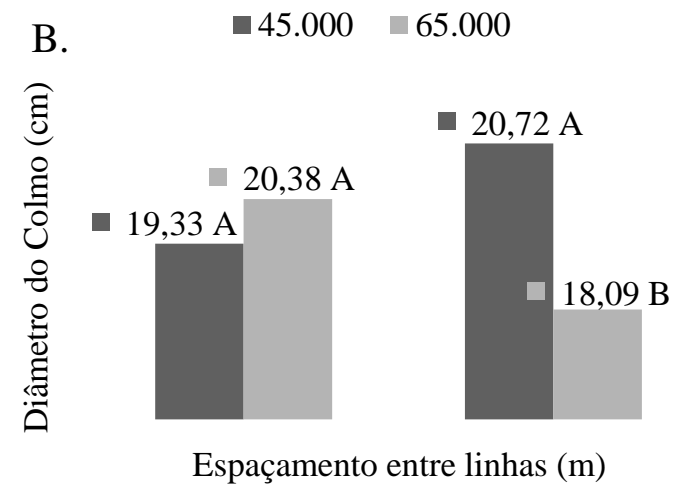

Figura 1. Desdobramento da interação para as médias do diâmetro do colmo, em função da população dentro do espaçamento (A) e espaçamento entre linhas dentro da população (B)

Ao analisar o espaçamento entre linha, observou-se que no espaçamento de $0,45 \mathrm{~m}$, o diâmetro do colmo não foi influenciado pela população. No entanto, o espaçamento de 0,90 $\mathrm{m}$, influenciou na população (Figura 2B), demonstrando que maiores populações, no menor espaçamento o diâmetro do colmo apresenta maior desenvolvimento. Isso pode ser considerada uma vantagem para a cultura, devido à resistência ao acamamento. Além de favorecer também, maior número de plantas na área, que poderá ou não, aumentar a produtividade pelo incremento de plantas na área em menores espaçamentos.

$\mathrm{Na}$ tabela 4 abaixo estão apresentados os valores médios para as variáveis de produção de milho. Observa-se que o índice de velocidade de emergência, número de espigas por hectare e produção por hectare; difere significativa para ambos os fatores (população de plantas e espaçamento). 
Tabela 4. Índice de velocidade de emergência (IVE), número de espigas por hectare (NEH), número de fileiras (NF), número de grãos por fileira (NGF) e produção por hectare (PH) em função da população de plantas e espaçamento entrelinhas na cultura do milho

\begin{tabular}{|c|c|c|c|c|c|}
\hline & $\begin{array}{l}\text { IVE } \\
(\%)\end{array}$ & $\begin{array}{c}\text { NEH } \\
\left(\text { espiga ha }^{-1}\right)\end{array}$ & NF & NGF & $\begin{array}{c}\mathrm{PH} \\
\left(\mathrm{kg} \mathrm{ha}^{-1}\right)\end{array}$ \\
\hline \multicolumn{6}{|c|}{ População (P) } \\
\hline 45.000 & $2,09 \mathrm{~b}$ & $24.777 \mathrm{~b}$ & 13,47 & 26,13 & $2.487 \mathrm{~b}$ \\
\hline 65.000 & 3,46 a & $36.500 \mathrm{a}$ & 13,23 & 24,45 & 3.438 a \\
\hline \multicolumn{6}{|c|}{ Espaçamento (E) } \\
\hline 0,45 & $2,47 \mathrm{~b}$ & $26.027 \mathrm{~b}$ & 13,40 & 25,96 & $2.597 \mathrm{~b}$ \\
\hline 0,90 & 3,08 a & 35.249 a & 13,31 & 24,60 & $3.328 \mathrm{a}$ \\
\hline \multicolumn{6}{|l|}{ Teste F } \\
\hline $\mathrm{P}$ & $29,18 * *$ & $103,69 * *$ & $0,47^{\mathrm{ns}}$ & $1,83^{\mathrm{ns}}$ & $12,51 * *$ \\
\hline $\mathrm{E}$ & $5,78 *$ & $64,18 * *$ & $0,06^{\mathrm{ns}}$ & $1,16^{\mathrm{ns}}$ & $7,40 * *$ \\
\hline $\mathrm{P} \times \mathrm{E}$ & $1,62^{\mathrm{ns}}$ & $1,12^{\mathrm{ns}}$ & $0,06^{\mathrm{ns}}$ & $0,11^{\text {ns }}$ & $0,14^{\mathrm{ns}}$ \\
\hline \multicolumn{6}{|l|}{ DMS } \\
\hline $\mathrm{P}$ & 0,50 & 2.293 & 0,68 & 2,51 & 535,45 \\
\hline E & 0,50 & 2.293 & 0,68 & 2,51 & 535,45 \\
\hline $\mathrm{CV}$ & 40,90 & 16,80 & 11,54 & 22,35 & 40,57 \\
\hline
\end{tabular}

${ }^{\mathrm{ns}}$ não significativo; ${ }^{*}$ significativo a 0,$05 ; * *$ significativo a 0,$01 ; \mathrm{CV}$ - coeficiente de variação

Para o fator população de plantas, a população de 65.000 plantas por hectare, apresentou média de produtividade superior quando comparada a população de 45.000. Este resultado pode ser atribuído ao aumento do número de espiga por hectare e, consequentemente, ao número de plantas por hectare. Tal resultado é bastante importante, pois mostra que o aumento da população de plantas favorece incremento em produtividade, o que nem sempre é observado no meio literário. Resultados semelhantes foram encontrados por Calonego et al. (2011).

Trabalhando com três espaçamentos entre fileiras e quatro populações, Fumagalli et al. (2017) concluíram que o aumento da população de plantas para a maioria dos componentes de rendimento da cultura do milho tem efeito positivo principalmente em espaçamentos reduzidos. Takasu et al. (2014) avaliando o desempenho agronômico do milho sob diferentes arranjos populacionais e espaçamentos entre linhas concluíram que a utilização do espaçamento de 0,45 metros entrelinhas proporciona aumento no número de grãos por fileira, no número de grãos por espiga, na massa de cem grãos e na produtividade de grãos na cultura do milho.
Assim como aconteceu para a população 65.000 plantas, maior produtividade, também foi encontrado maior produtividade no espaçamento de $0,90 \mathrm{~m}$. Resultados contrário foram encontrados por Modolo et al. (2010) ao observarem que os espaçamentos de 0,45 e 0,70 $\mathrm{m}$ proporcionaram resultados semelhantes, havendo queda no rendimento ao se utilizar o espaçamento de 0,90 m. Bem como Crusciol et al. (2012) no qual constataram que a produção por hectare é influenciada pela redução do espaçamento entre linhas, os quais, atribuíram tal resultado, a maior eficiência de interceptação da radiação e ao decréscimo de competição por recursos do meio, entre as plantas na linha.

\section{CONCLUSÕES}

1. A população de plantas e espaçamento influenciam os componentes da produção e a produtividade do milho.

2. A população de 65.000 plantas $h^{-1}$ proporciona incremento na produtividade de grãos, número de espigas por hectare, número de plantas por hectare e índice de velocidade de emergência, quando comparada com a população de 45.000 plantas ha ${ }^{-1}$.

3. O espaçamento de $0,90 \mathrm{~m}$ proporciona 
aumento na produtividade de grãos, número de espigas por hectare e índice de velocidade de emergência quando comparado com 0 espaçamento de $0,45 \mathrm{~m}$.

\section{LITERATURA CITADA}

ALBUQUERQUE, A. W.; SANTOS, J. R.; MOURA FILHO, G. E.; REIS, L. S. Plantas de cobertura e adubação nitrogenada na produção de milho em sistema de plantio direto. Revista Brasileira de Engenharia Agrícola e Ambiental, v.17, n.7, p.721-726, 2013. http://dx.doi.org/10.1590/S141543662013000700005

BRASIL. Ministério da Agricultura, Pecuária e Abastecimento. Regras para análise de sementes. Brasília, 2009. 395p.

CALONEGO, J. C.; POLETO, L. C.; DOMINGUES, F. N.; TIRITAN, C. S. Produtividade e crescimento de milho em diferentes arranjos de plantas. Agrarian, Dourados, v. 4, n.12, p. 84-90, 2011.

CONAB - Companhia Nacional de Abastecimento. Acompanhamento da safra brasileira de grãos 2015/2016. Décimo segundo levantamento. Disponível em:< $<$ http//www.conab.gov.br>. Acesso em: 21 nov. 2016.

CHIODEROLI, C. A.; MELLO, L. M. M.; GRIGOLLI, P. J.; FURLANI, C. E. A.; SILVA, J. O. R.; CESARIN, A. L. Atributos físicos do solo e produtividade de soja em sistema de consórcio milho e braquiária. Revista Brasileira de Engenharia Agrícola e Ambiental, v.16, n.1, p.37-43, 2012. http://dx.doi.org/10.1590/S1415-

43662012000100005

CRUSCIOL, C. A. C.; MATEUS, G. P.; NASCENTE, A. S.; MARTINS, P. O.; BORGHI, E.; PARIZ, M. An innovative copforage intercrops system: early cycle soybean cultivars and palisa degrass. Agronomy
Journal, v.104, n.4, p.1085-1095, 2012. http://dx.doi.org/10.2134/agronj2012.0002

DOORENBOS, J.; PRUITT, W. O. Crop water requirements. Rome: FAO Irrigation and drainage paper, 24, 1977. 154p.

EMBRAPA - Empresa Brasileira de Pesquisa Agropecuária. Manual de métodos de análise de solo. 2.ed. Rio de Janeiro: Embrapa Solos, 2011. 230p.

EMBRAPA - Empresa Brasileira de Pesquisa Agropecuária. Sistema brasileiro de classificação de solos. 3.ed. Brasília, 2013. 353p.

FANTIN, N. A. M.; MEERT, L.; HANEL, A.; ALENCAR, J. R. C.; PETEAN, L. P. Componentes de produção e qualidade de semeadura de soja em função de diferentes velocidades do conjunto trator-semeadora. Brazilian Journal of Applied Tecnology for Agricultural Science, v.9, n.3, p.7-15, 2016. (DOI): 10.5935/PAeT.V9.N3.01

FOLONI, J. S. S.; CALONEGO, J. C.; CATUCHI, T. A.: BELLEGGIA, N. A.; TIRITAN, C. S.; BARBOSA, A. M. Cultivares de milho em diferentes populações de plantas com espaçamento reduzido na safrinha. Revista Brasileira de Milho e Sorgo, v.13, n.3, p.312325, 2014.2 DOI: http://dx.doi.org/10.18512/1980-

6477/rbms.v13n3p312-325

FUMAGALLI, M.; MACHADO, R. A. F., FIORINI, I. V. A.; PEREIRA, C. S.; PIRES, L. P. M; PEREIRA, H. D. Desempenho produtivo do milho híbrido simples em função de espaçamentos entre fileiras e populações de plantas. Revista Brasileira de Milho e Sorgo, v.16, n.3, p. 426-439, $2017 . \quad$ DOI: http://dx.doi.org/10.18512/1980-

6477/rbms.v16n3p426-439

HOFFMANN, F. I.; JASPER, M.; SCREMIN, A. L. T. Avaliação de diferentes espaçamentos nos componentes de produção no milho (Zea mays L.). Revista da União Latino-americana 
de Tecnologia, Jaguariaíva, v. 2, n.3, p. 63-88, 2015.

KLEIN, J. L.; VIANA, A. F. P.; MARTINI, P. M.; ADAMS, S. M.; GUZATTO, C.; BONA, R. A.; RODRIGUES, L. S.; FILHO, D. C. A.; BRONDANI, I. L. Desempenho produtivo de híbridos de milho para a produção de silagem da planta inteira. Revista Brasileira de Milho e Sorgo, v.17, n.1, p. 101-110, 2018. DOI: http://dx.doi.org/10.18512/1980-

6477/rbms.v17n1p101-110

MODOLO, A. J.; CARNIELETTO, R.; KOLLING, E. M.; TROGELLO, E.; SGARBOSSA, M. Desempenho de híbridos de milho na Região Sudoeste do Paraná sob diferentes espaçamentos entre linhas. Ciência Agronômica, Fortaleza, v. 41, n.3, p. 435-441, 2010.

NEUMANN, M.; POCZYNEK, M.; LEÃO, G. F. M.; FIGUEIRA, D. N.; SOUZA, A. M. Desempenho de híbridos de milho para silagem cultivados em diferentes locais com três densidades populacionais. Revista Brasileira de Milho e Sorgo, v.17, n.1, p. 49-62, 2018. DOI: $\quad$ http://dx.doi.org/10.18512/19806477/rbms.v17n1p49-62

PORTO, A. P. F.; VASCONCELOS, R. C. DE; VIANA, A. E. S.; ALMEIDA, M. R. S. Variedade de milho a diferentes espaçamentos no Planalto de Vitória da Conquista - BA. Revista Brasileira de Ciências Agrárias, v.6, n.2, p.208-214, 2011. DOI:10.5039/agraria.v6i2a924
SANTOS, P. R. A.; CHIODEROLI, C. A.; LOUREIRO, D. R.; NICOLAU, F. E. A.; OLIVEIRA, J. L.P.; QUEIROZ, R. F. Características morfológicas e produtivas do milho no consórcio com forrageiras em diferentes épocas de semeadura. Revista Brasileira de Agricultura Irrigada, v. 11, n. 07, p. 2031-2039, $2017 . \quad$ DOI: 10.7127/rbai.v11n700678

SANGOI, L.; SILVA, P. R. F.; ARGENTA, G.; RAMBO, L. Ecofisiologia da cultura do milho para altos rendimentos. Lages: Graphel, 2010.

SILVA, A. F.; SCHONINGER, E. L.; CAIONE, G.; KUfFEL, C.; CARVALHO, M. A. C. Produtividade de híbridos de milho em função do espaçamento e da população de plantas em sistema de plantio convencional. Revista Brasileira de Milho e Sorgo, v.13, n.2, p. 162173, 2014.2 DOI: http://dx.doi.org/10.18512/1980-

6477/rbms.v13n2p162-173

SILVA, F. A. S.; AZEVEDO, C. A. V. Comparison of means of agricultural experimentation data through different tests using the software Assistat. African Journal of Agricultural Research, v.11, n.09, p. 35273531, 2016. DOI: 10.5897/AJAR2016.11523

TAKASU, A. T.; RODRIGUES, R. A. F.; GOES, R. J.; ARF, O.; HAGA, K. I. Desempenho agronômico do milho sob diferentes arranjos populacionais e espaçamento entrelinhas. Revista Agrarian, v.7, n.23, p.3441, 2014.2 DOI: https://doi.org/10.30612/agrarian.v7i23.2270 